\title{
THE APPLICABILITY AND EFFICIENCY OF THE ULTRASOUND EXAMINATION IN THE EVALUATION OF BONE HEALING PROCESS IN CHILDREN
}

\author{
Maria Daniela Trăilescu ${ }^{1,2}$, Adrian Ionel Pavel ${ }^{1,2}$, Camelia Doina Popescu ${ }^{3}$, Alexandru Mircea \\ Pop $^{1}$
}

\section{Abstract}

The introduction of minimally invasive osteosynthesis techniques in pediatric trauma requires the implementation of non-invasive methods of monitoring in the postoperative period until cure, new methods with no adverse effects on the young organism. The use of ultrasound in musculoskeletal pathology and traumatology has expanded rapidly over the last decade. While diagnostic ultrasound has been widely adopted in the field of soft-tissue pathology, ultrasound is not in routine use for fracture diagnosis or in the evaluation of bone healing process. Ultrasound examination of bone and periosteum is permissible using high frequency linear transducers. In addition, using power Doppler in the ultrasound examination of the musculoskeletal system can bring valuable local information about blood flow, which is important in assessing the favorable evolution of a fracture to heal.

Keywords: fractures, children, titanium elastic nails osteosynthesis, ultrasound, non-invasive assessment

\section{Introduction}

Although ultrasound is a fast, easy and long-used diagnostic imaging method in medical practice, for a long time it did not find its utility in orthopedics and traumatology, being considered useless due to the increased bone impedance, impenetrable by ultrasound. Precisely through this increased bone impedance, ultrasound is fully reflected at the bone surface, determining the hyperechoic appearance of the bone structure [1-4]. Ultrasound examination of the bone and periosteum is permissive by using linear transducers with a high frequency of 7.5-13 $\mathrm{MHz}$ Although the positive diagnosis of a fracture is established by radiography, ultrasound can reveal the fracture site, especially in children, where the periosteum takes off more easily, and the secondary hematoma is better represented as an interruption of the hyperechoic bone interface in case of fractures without displacement, or as an unevenness, more or less obvious, in the case of displacement fractures, consistent with the degree of displacement. In addition, the use of the power Doppler
Effect in the ultrasound examination of the musculoskeletal system can bring valuable local information related to blood flow, important in assessing the favorable evolution and establishing the prognosis of fracture healing [5-7].

\section{Purpose}

The primary objectives of our research are to assert the use and validity of musculoskeletal ultrasound as a noninvasive imaging method for identifying and evaluating bone callus in the dynamics of the post interventional bone healing process, as well as demonstrating particular aspects of the evolution to cure the fracture site stabilized with titanium elastic nails. Ultrasounds performed at the level of a fracture site stabilized by minimally invasive osteosynthesis were performed easily, away from the place of insertion of osteosynthesis materials, and implicitly away from the surgical wound. The ultrasound examination maneuver, which aimed to examine the fracture site in the transverse and longitudinal plane, did not cause additional discomfort to the patients. By centromedullary placing of the elastic titanium nails, the fracture site is viewable during ultrasound, there is no interposition between the site fracture and the linear transducer.

We chose the following intervals of ultrasound examination of the fracture site according to the three important stages of indirect bone healing, specific to the chosen orthopedic surgery technique, during which ultrasound examination of local vascularization is a predictive factor for favorable or unfavorable prognosis of fracture healing. These are represented by the cell proliferation stage, the fibrous callus stage and the consolidation stage. We excluded the hematoma phase from the first 7 days and the remodeling phase that occurs after 30 days because the vascularization adjacent to the fracture site is poorly represented.

The musculoskeletal ultrasound examination and the Doppler ultrasound examination of the fracture site was performed by the same operator, with the same ultrasound device, thus removing the subjective factor, dependent on the examining physician.

${ }^{1}$ Department of Pediatric Surgery and Orthopedics, Emergency County Hospital, Arad, Romania

${ }^{2}$ Faculty of Medicine, "Vasile Goldiş" Western University of Arad, Romania

${ }^{3}$ Radiology Pediatric Department, Emergency County Hospital, Arad, Romania

E-mail: trailescumaria@yahoo.com, adipave172@yahoo.com, camidoinap@yahoo.com, alexanpop@yahoo.com 
Table I. Results of the ultrasound and power Doppler study of the fracture site

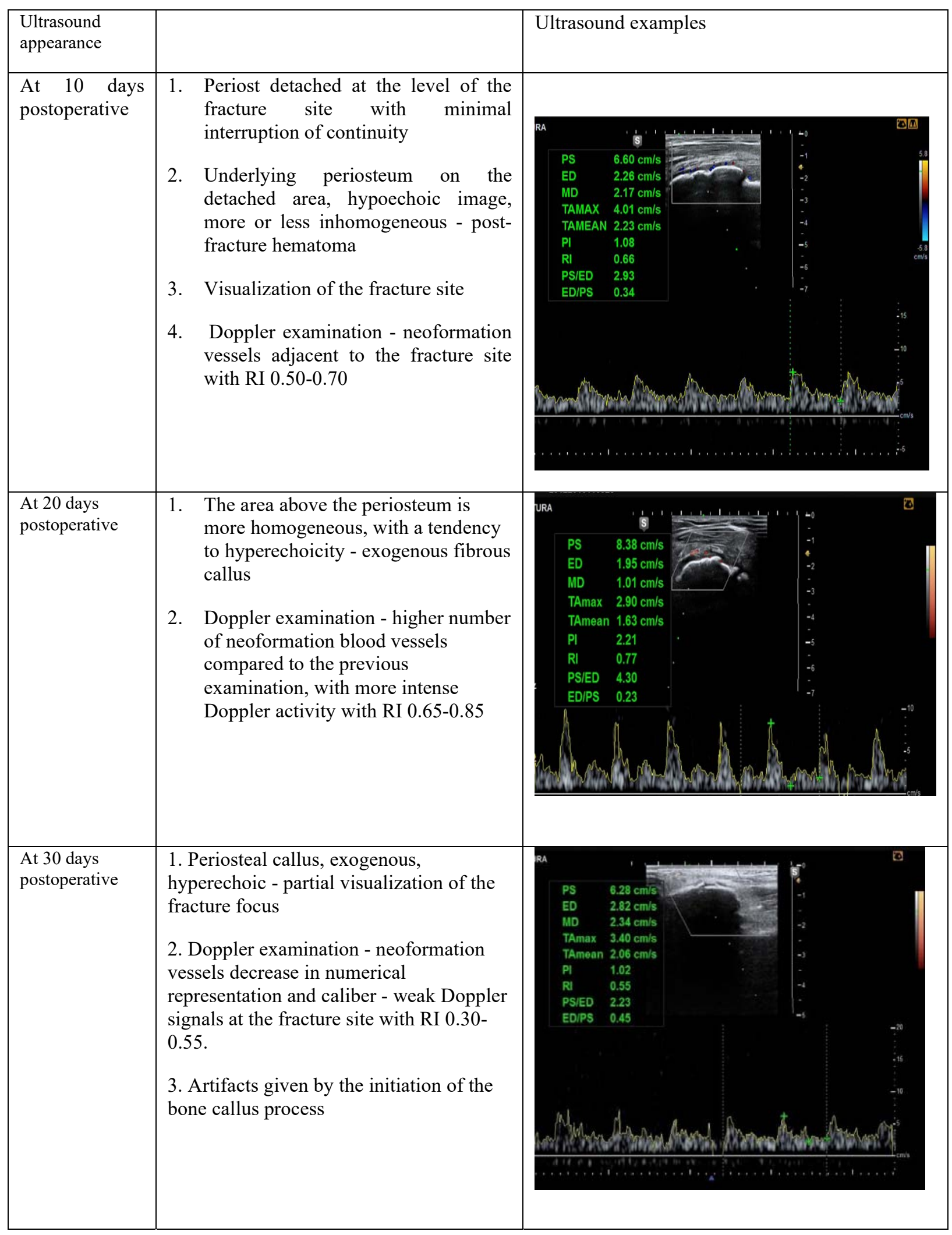


The results of the fracture site obtained by ultrasound examination of the fracture site performed at 30 days was compared with the radiological examination of the fracture site performed at the same postoperative time interval, as a radiography is the universally recommended method of monitoring fracture healing one month after the surgery.

\section{Material and methods}

This prospective study was held over a three-year period from 2015 until 2018 covering 167 children aged 5 to 18 years, admitted to the Department of Pediatric Surgery and Orthopedics, Emergency County Hospital, Arad, Romania with therapeutic indication for osteosynthesis for displaced diaphysis fractures of the humerus, forearm, femur and tibia.

In all cases, we practiced minimally invasive osteosynthesis with titanium elastic nails (TEN). The ultrasound examination was performed with a Samsung UGEO H60 ultrasound device, equipped with musculoskeletal software, power Doppler and a linear LA314AD transducer with a frequency of 7-13 MHz

Patients were monitored postoperative by ultrasound at the established time intervals, respectively at 10 days, 20 days and 30 days. During ultrasound examination measurements of the callus were performed. Using the power Doppler callus vascularity was visualized and vascular resistance index (RI) was measured.

The results obtained by ultrasound at 30 days postoperative were compared with radiograph measurements and with the assessment of the callus quality.

\section{Results}

At 10 days after osteosynthesis, musculoskeletal ultrasound clearly reveals the fracture site, the distance between the fracture fragments, the more or less hypoechoic local hematoma, and the degree of periosteal detachment. Doppler examination identifies the appearance of neoformation vessels adjacent to the interrupted periosteum. The visualization of neoformation vessels, with a fine linear appearance on longitudinal sections, or with a punctiform appearance on cross sections, represents a favorable prognostic factor for fracture healing -Table I. Doppler determination of vascular resistance index values in neoformation vessels involves patience and extra time, as the patient's movement can yield numerous artifacts. The values of the vascular resistance index measured at this time were between $0.50-0.70$, with differences depending on the ultrasound explored segment.

At 20 days after osteosynthesis, musculoskeletal ultrasound clearly detects the fracture site, the distance between the fracture fragments, more or less hyperechoic, more organized exogenous fibrous callus, thickened periosteum and hyperechoic. Doppler examination identifies neoformation vessels, better represented numerically and with a larger caliber than in the previous examination, adjacent to the better visible periosteum. The values of the vascular resistance index measured at this time were between $0.65-0.80$.
At 30 days after osteosynthesis, musculoskeletal ultrasound partially detects the fracture site, but between the fracture ends and their overlying is the presence of bone callus, as a hyperechoic bridge, irregular, measurable in length and thickness by clear delimitation of adjacent soft tissues. Doppler examination identifies poorly represented and smaller caliber neoformation vessels than in the previous examination. The values of the vascular resistance index measured at this time were between $0.30-0.55$.

The comparative ultrasound and radiological studies of the fracture site performed at 30 days identifies the presence of the periosteal callus. On an ultrasound, periosteal callus, exogenous, hyperechoic allows partial visualization of the fracture site and osteosynthesis material.

Radiologically, the appearance of unstructured, partially mineralized osseous callus is identified, with the vague visualization of the fracture line, which still remains clearly detectable. The appearance of the callus has a lower color intensity than normal bone tissue.

The comparative studies, at 30 days postoperative, of the results of the two imaging examinations confirmed the ultrasound detection of the callus in $95 \%$ of the examined cases, while the radiological examination showed the callus in $74 \%$ of the cases - Fig. 1 .

The analysis of the cases in which the callus was identified by the two methods, respectively $74 \%$ of cases, clearly showed that musculoskeletal ultrasound is an imaging method clearly superior to radiography, by earlier identification and much more accurate callus measurement, the dimensions of the callus measured ultrasound showing values 1.5-2 $\mathrm{mm}$ larger than those determined radiologically in all cases - Fig. 2 .

\section{Discussions}

Although there are treatises or atlases of musculoskeletal ultrasound in which there are descriptions of recent fractures, in current practice the positive diagnosis of fracture is commonly established by radiological examinations, the few existing studies in the literature bringing discordant data about false negative or positive ultrasound diagnosis results.

The use of ultrasonography in bone evaluation raised and still raises a lot of controversies.

Ultrasound allows to show only the outer surface of the bone, with no access to the trabecular bone. Moreover, not every area of the bone is available to ultrasound [8].

Despite these major disadvantages the publications show a high correlation of ultrasonography with radiographs in the diagnosis of sternum and long bone fractures, as well as in the evaluation of fractures in children $[9,10]$.

Based on the research, it was found that vascular resistance and vessel density in the callus are greatest during the initial healing phase and gradually decrease from the 3-4 week after the fracture.

Analysis of the results showed a high value of ultrasonographic evaluation of the vascularity of the callus in predicting normal or delayed healing $[11,12]$. 


\section{Conclusions}

Through the intramedullary assembly of the nails, the fracture site is easy to examine by ultrasound, the applicability being demonstrated by the present study, while the possibility of ultrasound monitoring of the fracture focus minimally invasive is another indirect advantage of the surgical technique.

Ultrasound examinations with a high frequency linear transducer allowed me to clearly identify in the first phase the fracture focus, the post-fracture hematoma and the periosteum, then the fibrous callus at 20 days and the bone callus at 30 days, measurable in cross section and longitudinally.
Ultrasound examinations in dynamics corresponded to the histological phases of bone healing, the Doppler Effect faithfully capturing the neovascularization phases, respectively their appearance, progression and regression. The values of the resistance index of the neoformation vessels adjacent to the fracture site confirmed the neovascularization phases by the evolution of the values during the examinations. The values recorded were directly dependent on the time of examination, the segment examined and the age of the patient, explaining the variation of the values of the vascular resistance index during the examinations.

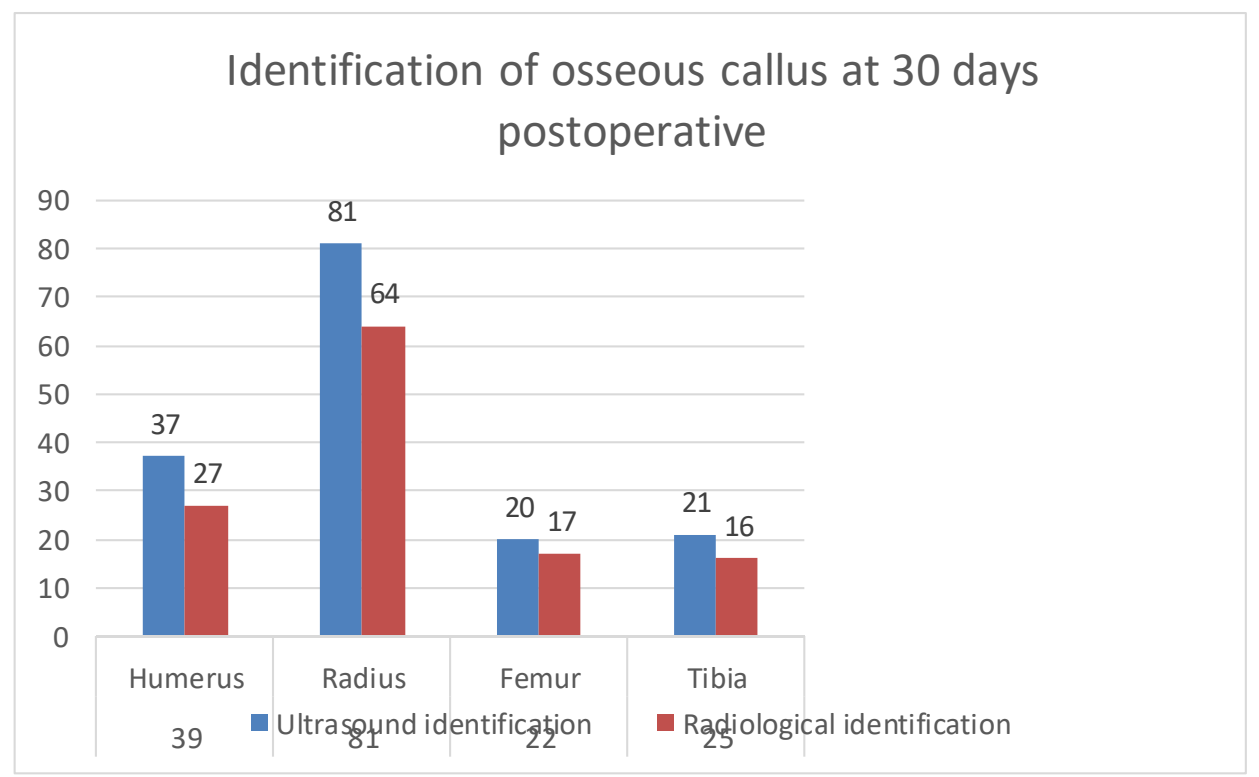

Fig. 1. Comparative identification of the osseous callus by the two imaging methods 


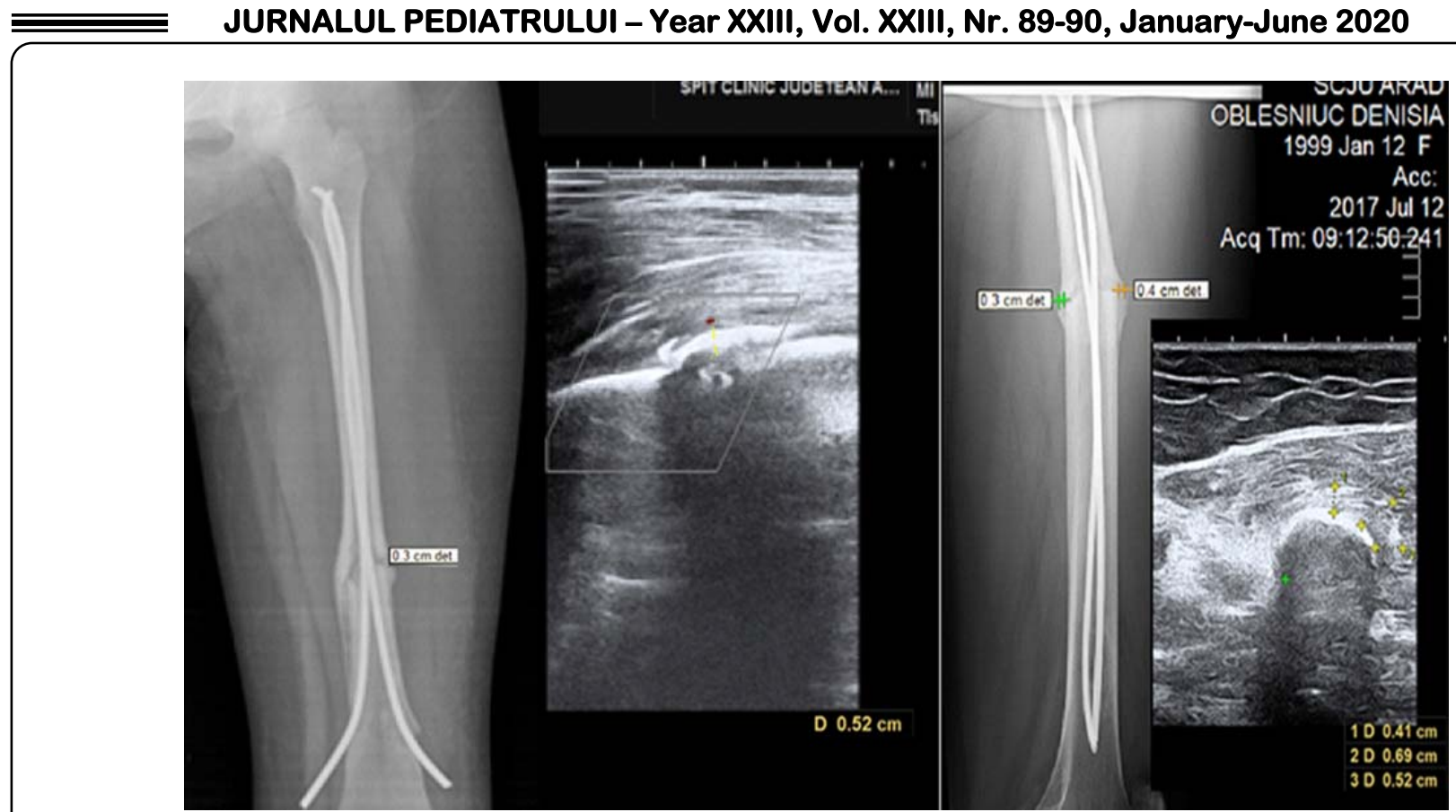

Fig. 2. Comparative radiological imaging aspects (AP and LL incidence) and ultrasound (longitudinal and cross section) of the bone callus at 30 days postosteosynthesis with TEN, femoral shaft fractures.

Identifying and measuring the fibrous and bony callus via ultrasound presents a real advantage for monitoring and assessing the progression to healing of a fracture, ultrasound of the fracture focus performed at 30 days identifying callus formation in $95 \%$ of cases, compared to radiography that confirmed the presence of callus only in $74 \%$ of cases.

Ultrasound examination requires experience, time, tact and collaboration with small patients. This is particularly important in Doppler examinations, where minor movements limb cause artifacts and, implicitly, an erroneous result. The repeatability and dynamics of the ultrasound allows for a more frequent examination of the same patient throughout the healing process. In the presence of clinical signs of favorable evolution, musculoskeletal ultrasound is a non-invasive imaging method, safe and can be used to monitor the healing of a fracture.

Knowledge of the peculiarities of immature bone and the physiological process of fracture healing in children, knowledge of minimally invasive osteosynthesis technique with elastic titanium rods, radiological and ultrasound evolution of the fracture site, gives the pediatric orthopedist the chance to ultrasound monitor the evolution of the healing fracture, provided that the relevant knowledge of musculoskeletal ultrasound is assimilated.

\section{References}

1. Fodor D. Ecografie clinică musculoscheletală. Editura Medicală. 2009, 46-47.

2. Eckert K, Ackermann O, Schweiger B, Radelff E, Liedgens $\mathrm{P}$. Ultrasound as a viable alternative to standard X-rays for the diagnosis of distal forearm fracture in children. Z Orthop Unfall. 2012, 150(4): 409- 14.

3. Mortiz JD, Berthold LD, Soenksen SF, Alzen GF. Ultrasound in diagnosis of fracture in children: Unnecessary harassment or useful addition to X-ray? Ultraschall Med. 2008, 29(3): 267-74.

4. Barata L, Spencer R, suppiah A, Raio C, Ward MF, Sama A. Emergency ultrasound in the detection of pediatric long-bone fractures. Pediatr Emerg Care. 2012, 28(11):1154-7

5. Hübner U, Schlicht W, Outzen S, Barthel M, Halsband $\mathrm{H}$. Ultrasound in the diagnosis of fractures in children. $\mathrm{J}$ Bone Joint Surg Br. 2000, 82(8):1170-3.

6. Bottinelli O, Calliada F, Campani R. Bone callus: possible assessment with color Doppler ultrasonography. Normal bone healing process. Radiol Med. 1996, 91(5):537-41.

7. Caruso G, Lagalla R, Derchi L, Iovane A, Sanfilippo A. Monitoring of fracture calluses with color Doppler sonography, J Clin Ultrasound. 2000, 28(1): 20-27.

8. Magdalena Wawrzyk, Jan Sokal, Ewa Andrzejewska, Przemysław Przewratil. The Role of Ultrasound Imaging of Callus Formation in the Treatment of Long Bone Fractures in Children. Pol J Radiol. 2015, 80: 473-478. 
9. Patten RM, Mack LA, Wang KY, Lingel J. Nondisplaced fractures of the greater tuberosity of the humerus: sonografic detection. Radiology. 1992, 182, 201-4.

10. Rathfelder FJ, Paar O. Possibilities for using sonography as a diagnostic procedure in fractures during the growth period. Unfallchirurg. 1995, 98: 64549.
11. Caruso G, Lagalla R, Derchi L et al: Monitoring of fracture calluses with color Doppler sonography. J Clin Ultrasound. 2000, 28(1): 20-27.

12. Risselada M, Kramer M, Saunders JH et al: Power Doppler assessment of the neovascularization during uncomplicated fracture healing of long bones in dogs and cats. Vet Radiol Ultrasound. 2006, 47(3): 301-6

Correspondence to:

Maria Daniela Trăilescu

Department of Pediatric Surgery and Orthopedics,

Emergency County Hospital, Arad, Romania

Andreny Karolly Street, No.2 - 4,

Arad, 310037, România

Phone: +40-257- 220000

Email: trailescumaria@yahoo.com 